\title{
Inhaltsverzeichnis
}

I. Einleitung - Jörg Dünne und Andreas Mahler — 1

II. Modelle und Theorien

II.1 Achsen

1. Topologie - Andreas Mahler -17

2. Topographien: Zur Ausgestaltung literarischer Räume Wolfram Nitsch $\mathbf{3 0}$

3. Dynamisierungen: Bewegung und Situationsbildung Jörg Dünne — 41

\section{II.2 Räume}

4. Deixis - Michael Cuntz $\longrightarrow \mathbf{5 7}$

\section{FELDER}

5.

Schrifträume - Hermann Doetsch — 73

6. Der literarische Raum - Xavier Garnier — 88

7. Raum und Erzählung - Birgit Neumann — 96

8. Raum und Theatralität - Sabine Friedrich $-\mathbf{1 0 5}$

9. Räume des Wissens - Burkhardt Wolf — $\mathbf{1 1 5}$

10. Geopolitik und Globalisierung - Niels Werber - 126

11. Postkoloniale Räume - Tobias Döring - 137

\section{ORTE}

12

Landschaft - Michel Collot -151

13. Chronotopoi - Michael C. Frank - 160

14. Semiosphäre und Sujet - Cornelia Ruhe - $\mathbf{1 7 0}$

15. Utopie und Heterotopie - Rainer Warning - $\mathbf{1 7 8}$ 
16. Nicht-Orte - Matei Chihaia $\mathbf{1 8 8}$

17. Mnemotop - Nicolas Pethes - 196

\section{WEGE}

18. Ecocriticism und Geopoetik - Tatjana Hofmann — 207

19. Literarische Geographie und Geokritik - Michel Collot -217

20. Literaturgeographie und Literaturkartographie Barbara Piatti — 227

21. Literarisches Feld - Joseph Jurt $-\mathbf{2 4 0}$

22. Kartographisches Schreiben und kartographische Imagination Federico Italiano $-\mathbf{2 4 9}$

23. Transitorische Räume - Vittoria Borsò $\mathbf{2 5 9}$

24. Nicht-euklidische Räume - Oliver Simons $-\mathbf{2 7 2}$

III. Paradigmen

\section{III.1 Plateaus}

25. Formationen literarischer Raumgeschichte - Roger Lüdeke $-\mathbf{2 8 5}$

\section{III.2 Texträume und Raumtexte}

26. Das Mittelmeer: Reisen, Navigieren, Erzählen Markus Janka - 301

27. Athen: Autochthoner Raum und politisches Theater Susanne Gödde - 312

28. Rom: Imperium Romanum - Bernhard Teuber - 324

29. Vom Artushof nach King's Landing: Chrestien de Troyes und die Mediävisierung politischer Topographie - Xuan Jing - 335

30. Der lange Raum: Die grosse kirch in der Vnderweisung der kunst des Messens der Offizin Hieronymus Rodler - Christina Lechtermann 344

31. Die ,Neue Welt‘: Reisen und Alterität - Hanno Ehrlicher — 355 
32. Atlantik/Pazifik: Die imaginäre Erschließung der Ozeane im Zeitalter der Segelschifffahrt - Margaret Cohen - 364

33. Venedig: Ambiguität der Republik - Isabel Karremann - $\mathbf{3 7 6}$

34. London: Frühneuzeitliche Großstadt - Sabine Schülting - $\mathbf{3 8 5}$

35. Die Straße: Mobilität und Festkultur im Siglo de Oro - Miriam Lay Brander - 393

36. Versailles: Höfische Spielräume - Ulrike Sprenger - 403

37. Das Kaffeehaus: Bürgerliche Öffentlichkeit - Sebastian Domsch - 413

38. Nissopoiesis: Wie Robinsone ihre Inseln erzählen - Daniel Graziadei - 421

39. Paris: Urbanität, Entgrenzung, Flüchtigkeit - Karin Westerwelle - 431

40. Meer/Luft/Wüste: Eroberung des Naturraums - Wolfgang Struck -442

41. Intervenierendes Papier': Die Seite als Schrift- und Denkraum Kurt Hahn - 451

42. St. Petersburg: Exzentrische Moderne - Susi K. Frank - 461

43. New York: „The center of things“Die Raumzeit der Metropole - Johanna Schumm — 469

44. Tlön: Imaginationsräume und Ander-Welten - Victor $A$. Ferretti- 478

45. Das Lager: Raum der Ausnahme - Judith Kasper - 485

46. Megastadt: Kinematographisches Mumbai und das Rhizom des Abjekten - André Otto - 494

47. Korallen: Migration und Transozeanität - Johanna Abel und Gesine Müller - $\mathbf{5 0 5}$ 
VIII _ Inhaltsverzeichnis

IV. Glossar $-\mathbf{5 1 5}$

V. Auswahlbibliographie $-\mathbf{5 2 9}$

VI. Register

Personenregister $-\mathbf{5 6 3}$

Sachregister $\mathbf{-} \mathbf{5 7 1}$

VII. Abbildungsnachweise $-\mathbf{5 8 5}$

VIII. Autorinnen und Autoren $-\mathbf{5 8 7}$ 